\title{
Análisis de la reproducibilidad y repetitividad en ensayos reológicos I suspensiones de óxido de silicio y etilenglicol
}

\author{
L.F. Naranjo Herrera ${ }^{\mathrm{a}}$, F.J. Rubio Hernández ${ }^{\mathrm{b}}$, R. Delgado García ${ }^{\mathrm{a}}$, N.M. Páez Flor ${ }^{\mathrm{ab}}$, C.N. Villacís Núñez ${ }^{\mathrm{a}}$, J.G. Melo \\ Altamirano $^{\mathrm{a}}$ \\ ${ }^{a}$ Departamento de Ciencias de la Energía y Mecánica, Universidad de las Fuerzas Armadas ESPE, Ecuador \\ ${ }^{\mathrm{b}}$ Departamento de Física Aplicada II, Universidad de Málaga, España \\ Ifnaranjo4@espe.edu.ec, fjrubio@uma.es, rrdelgado1@espe.edu.ec,nmpaez@espe.edu.ec, cnvillacis@espe.edu.ec,
} jgmelo@espe.edu.ec

\begin{abstract}
Resumen-El presente estudio tiene como principal objetivo encontrar un protocolo adecuado para la experimentación repetible y reproducible de ensayos reológicos de suspensiones con nanopartículas. Se consideraron mezclas con diferentes concentraciones de óxido de silicio y etilenglicol para el análisis. Se experimentó inicialmente con control de velocidad en una geometría cono - plato, para posteriormente cambiar a control de esfuerzo en una geometría de vanos. Se observó el comportamiento reológico con saltos de escalón en búsqueda de reproducibilidad. También se comparó los resultados obtenidos con y sin aplicación de baño ultrasónico para homogeneización de la suspensión. Finalmente, se compararon las gráficas construidas $y$ se definieron los parámetros adecuados para garantizar reproducibilidad en este tipo de suspensiones.
\end{abstract}

Palabras Claves-Reología, reproducibilidad, suspensión

Abstract-The goal of this study is to find an appropriate protocol to develop repeatable and reproducible rheological experimentation of suspensions with nanoparticles. Mixtures with different concentrations of silicon dioxide and ethylene glycol were considered for analysis. Initially, shear rate control and cone plate geometry were used; then, the conditions changed to stress control and vane geometry. The rheological behavior was observed by applying steps in order to find reproducibility. Results with and without ultrasonic bath were also compared. Ultrasonic bath was applied to homogenize the samples. Ultimately, the resulting graphs were compared and the appropriate parameters to guarantee reproducibility in these kinds of suspensions were defined.

Keywords— Rheology, reproducibility, suspension

\section{INTRODUCCIÓN}

Una suspensión se puede definir como una mezcla heterogénea (microscópicamente, ya que macroscópicamente es homogénea) entre un líquido (fase dispersante) y partículas sólidas no solubles (fase dispersa). Son varios los estudios que han tratado de verificar la relación existente entre la reología de suspensiones y su microestructura [1], [2].

Wei-Guo y Struble [3], estudiaron el comportamiento reológico de la pasta de cemento fresca a través de técnicas de microscopía, concluyendo que, durante el periodo de inducción, el incremento del esfuerzo de fluencia es el resultado de la acumulación de gel entre los granos de cemento, lo que, a su vez, aumenta la fuerza de cohesión entre los granos, infiriendo directamente sobre su viscosidad. Stickel y Powell [4] determinaron que la descripción de la microestructura resulta compleja dado que es necesario tener datos de la posición exacta de todas las partículas; sin embargo, pudieron obtener un modelo para migración de partículas por efecto de la velocidad de cizalla.

La concentración de partículas y la intensidad de las fuerzas con las que interactúan influyen en dos características de las suspensiones de partículas. En primer lugar, dependiendo de las fuerzas de repulsión y atracción entre las partículas, la suspensión estará más o menos estructurada lo que determinará si su viscosidad aumenta o disminuye en función de la velocidad de cizalla impuesta. De manera general, si las fuerzas de atracción son predominantes, la suspensión tiende a flocular cuando la cizalla tiende a cero (shear-thinning); si las fuerzas de repulsión predominan, la suspensión puede dar lugar a la formación de agregados de partículas cuando la cizalla aumenta (shear-thickening). En segundo lugar, las partículas sólidas podrían flotar en la superficie, suspenderse o sedimentar, dependiendo de la densidad de cada una de las fases, de la temperatura, de la morfología de las partículas así como también de las fuerzas de atracción-repulsión entre ellas.

Encontrar un procedimiento adecuado para realizar medidas reológicas de suspensiones de partículas, tomando en cuenta las interacciones entre ellas, es la razón principal que motiva el presente estudio; así como la proyección de futuras aplicaciones asociadas [5]-[10]. Concretamente, en el siguiente artículo se describe detalladamente el protocolo de preparación de suspensiones de óxido de silicio en etilenglicol y el análisis de la reproducibilidad y repetitividad de determinados ensayos reológicos.

\section{MATERIALES Y MÉTODOS}

\section{A. Materiales y equipos}

En este estudio se trabajó con suspensiones de óxido de silicio (como fase sólida) y etilenglicol (como fase líquida). Las nanopartículas de SiO2 (US Research Nanomaterials Inc.) tienen tamaños de partícula comprendidos entre $60 \mathrm{y}$ $70 \mathrm{~nm}$, pureza del 98\% y forma amorfa. El etilenglicol (Loba Chemie) es de grado analítico y tiene una pureza mínima del 99.5\%. Los ensayos reológicos se llevaron a cabo en un reómetro de esfuerzo controlado Discovery HR2 (TA-Instruments) del laboratorio de Reología de la Universidad de las Fuerzas Armadas ESPE. Las suspensiones se prepararon con la ayuda de un agitador 
magnético S130815 (Thermo Scientific), y un baño ultrasónico digital PS-40A (AionScientic) de $40 \mathrm{kHz}$. Para verificar la reproducibilidad de los resultados se utilizó un reómetro Haake MarsIII (Thermo Scientific) del laboratorio de Reología de la Universidad de Málaga. Las geometrías utilizadas en ambos casos fueron cono-plato y de vanos.

\section{B. Protocolo de preparación de muestras}

Para la preparación de muestras de suspensiones de óxido de silicio y etilenglicol se realizó una mezcla w/w. Primero se colocó la fase sólida en un frasco de plástico y, posteriormente, la fase líquida, sellando el frasco para evitar que la muestra reaccione con el medio ambiente. Posteriormente, se aplicó agitación magnética con velocidad de rotación estable por 120 minutos a temperatura ambiente. Finalmente, se aplicó a la muestra ultrasonidos durante 30 minutos a temperatura ambiente. El proceso de ultrasonido reduce la polidispersidad de la fase sólida.

\section{Experimentación}

Una vez preparada la suspensión, se la llevó al reómetro y se realizaron ensayos de salto de escalón con control de velocidad, usando en primer lugar la geometría cono-plato (cono de 60mm de diámetro y ángulo de 2 grados). Para cada experimento se aplicó una pre-cizalla inicial, con la que se eliminaron respuestas erráticas resultantes de la influencia de la historia de cizalla en la respuesta reológica del material. Adicionalmente, el estudio reológico se realizó partiendo de un misma microestructura inicial.

Como se mostrará más adelante, los ensayos no presentaron repetitividad. Asumiendo que esto podría deberse a sedimentación de una parte de la fase sólida, se procedió a realizar pruebas con una geometría capaz de reducir parcialmente el efecto de la sedimentación (cilindros concéntricos). Más específicamente, se decidió trabajar con una geometría de vanos (diámetro $28 \mathrm{~mm}$ y longitud $42 \mathrm{~mm}$ ) para reducir deslizamientos en las paredes del rotor. Para evitar posibles errores debido a que el equipo tenga dificultad en mantener velocidades de cizallas pequeñas, estos ensayos se realizaron en modo de control de esfuerzos.

Para obtener el valor máximo de cizalla previo a la turbulencia (las condiciones básicas para estudios reológicos son: flujo laminar, minimizar cambios físicos, reproducibilidad y repetitividad) se analizó las expresiones (1) y (2). La expresión 2 es propia de un fluido newtoniano pero se la puede usar para una cizalla específica. Luego, se despejó el esfuerzo de cizalla y se analizó en las condiciones en las que sería crítico como se muestra en la expresión (3). De todas maneras otro limitante en la cizalla máxima es que el material se mantenga entre las geometrías del reómetro y obviamente que el reómetro sea capaz de aplicar este esfuerzo de cizalla.

$$
\begin{gathered}
\dot{\gamma}=\frac{\operatorname{Re} \cdot \eta}{\rho \cdot D} \\
\tau=\dot{\gamma} \cdot \eta
\end{gathered}
$$

Al sustituir (2) en (3) se obtiene:

$$
\tau=\frac{\operatorname{Re} \cdot \eta^{2}}{\rho \cdot D} \rightarrow \tau_{\text {cizalla maxima }}=\frac{2000 \cdot \eta^{2}}{\rho \cdot D}
$$

\section{ANÁLISIS DE RESUlTADOS}

\section{A. Saltos de Escalón con control de velocidad}

Se realizaron ensayos en modo de control de velocidad a concentraciones de $\mathrm{SiO}_{2}$ entre 10 y 30\% w/w. Para soluciones al $10 \%$ de $\mathrm{SiO}_{2}$, la desviación existente entre un ensayo y otro fue menor al 10\%, Fig. 1.

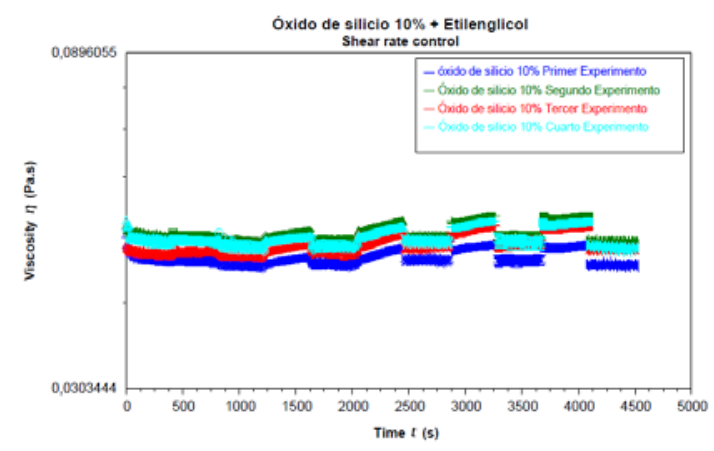

Fig. 1. Saltos de Escalón con control de velocidad $\mathrm{SiO}_{2} 10 \% \mathrm{w} / \mathrm{w}$

$\mathrm{Al}$ aumentar la concentración del $\mathrm{SiO}_{2}$ al $20 \%$ se muestra un dispersión mucho mayor, en lugar de traslaparse los resultados de viscosidad, su valor disminuye secuencialmente de un ensayo a otro, Fig. 2. A partir de esta concentración se puede observar que los valores estacionarios de viscosidad, a distintos cambios de velocidad de cizalla, varían notablemente a diferencia de los resultados al $10 \%$ de concentración. Esto indica que la respuesta es no-newtoniana y que la viscosidad depende de la velocidad de cizalla impuesta. Aunque cada experimento varía considerablemente uno de otro, la respuesta tiene la misma tendencia. Las respuestas son cada vez menos viscosas y existen cambios de velocidad de cizalla que no llegaron al valor estacionario.

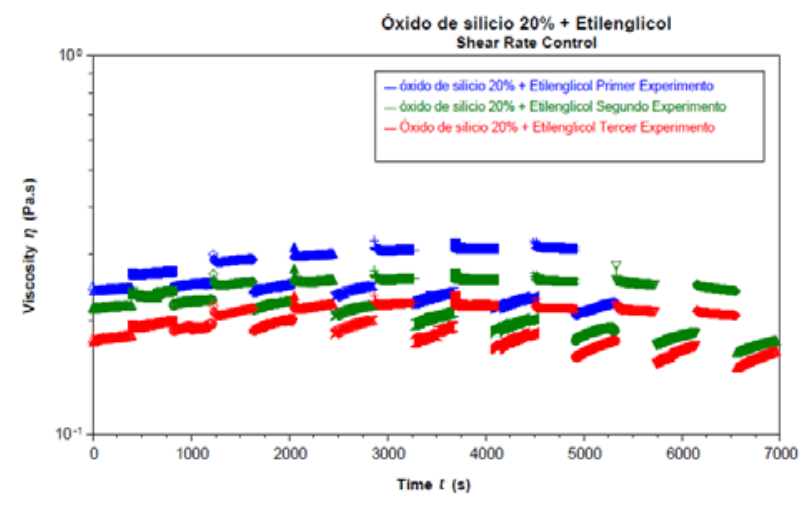

Fig. 2. Saltos de Escalón con control de velocidad $\mathrm{SiO}_{2} 20 \%$ w/w.

Al aumentar la concentración al 30\% w/w se observa una disminución progresiva en la viscosidad tanto al aumentar la velocidad de cizalla a lo largo del experimento como también en los experimentos sucesivos. El estado estacionario para cada velocidad de cizalla no pudo ser alcanzado, lo cual evidencia la falta de estabilidad en la suspensión producto de un proceso de sedimentación que es mayor cuando la concentración aumenta, Fig. 3. Por esta razón, se decidió no seguir con la experimentación utilizando la geometría cono-plato. Para verificar la reproducibilidad se llevó a cabo algunos de los experimentos mencionados en otro Reómetro (Haake 
MarsIII) en el Laboratorio de Reología de la Universidad de Málaga, como indica la Fig. 4. Los resultados presentan reproducibilidad pero no repetitividad. La respuesta viscosa varía en función de la velocidad de cizalla y disminuye para cada nuevo ensayo. Se videncia que la estabilidad de la suspensión presenta un problema de sedimentación. Esta comparación se la realizó únicamente con concentraciones al $20 \%$ de fase sólida.

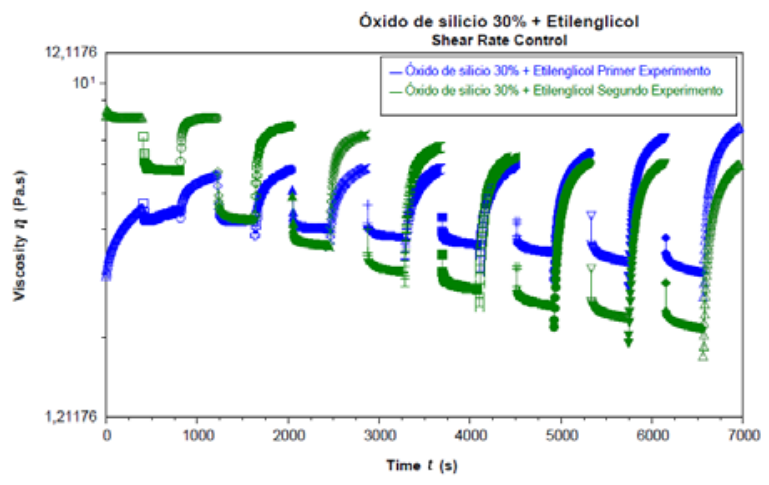

Fig. 3. Saltos de Escalón con control de velocidad $\mathrm{SiO}_{2} 30 \% \mathrm{w} / \mathrm{w}$

\section{B. Saltos de Escalón con control de esfuerzos}

Dado que en ningún experimento, utilizando el modo de control de velocidad con geometría cono-plato, se pudo obtener repetitividad en los ensayos, se realizaron experimentos en modo de control de esfuerzos utilizando una geometría de vanos. El modo de control de esfuerzos se utilizó para verificar si el reómetro tenía inconvenientes en mantener la velocidad de cizalla impuesta. La geometría de vanos se utilizó para disminuir el efecto de sedimentación de las partículas en suspensión y evitar posibles efectos de deslizamiento. La solución al 20\%w/w mostró estabilidad en la ejecución de los experimentos y presentó un comportamiento ligeramente no-Newtoniano.

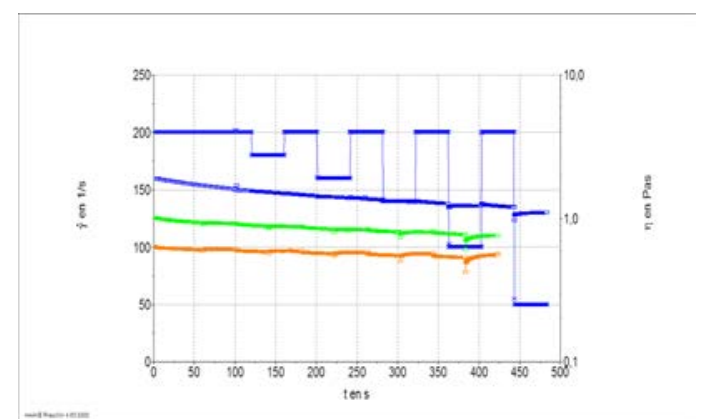

Fig. 4. Saltos de Escalón con control de velocidad $\mathrm{SiO}_{2} 20 \%$ w/w, Reómetro Haake MarsIII. La respuesta azul es la primera seguida por la verde y finalmente por la anaranjada.

Se consiguió una repetitividad aceptable, la cual se evidencia en la forma en la que las gráficas se sobreponen, con variaciones menores al 5\%, Fig. 5. En la Fig. 6 se procedió a repetir las medidas de la Fig. 5 desde la preparación de la muestra, por otro operador y aumentando el tiempo de la pre-cizalla para verificar la reproducibilidad. Como se observa en la figura en mención, los resultados son reproducibles. Mientras mayor era el esfuerzo de pre-cizalla se necesitó mayor tiempo para alcanzar el valor estacionario, por lo cual se optó por trabajar con una pre-cizalla más baja.

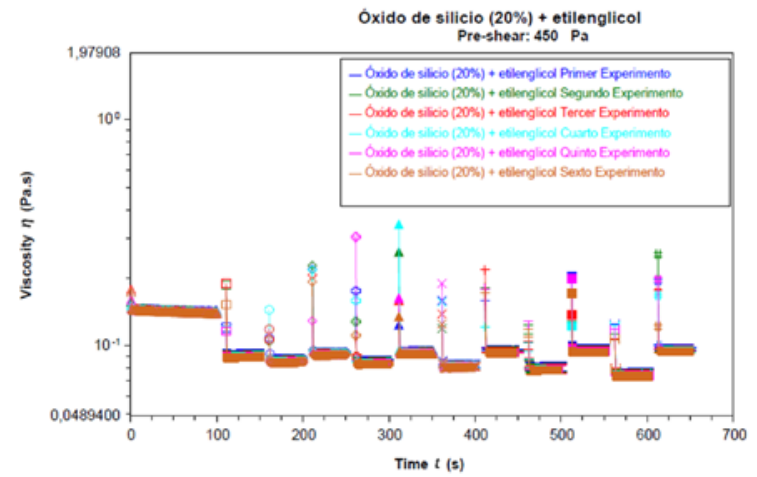

Fig. 5. Saltos de Escalón con control de esfuerzos de $\mathrm{SiO}_{2} 20 \%$ w/w con $450[\mathrm{~Pa}]$ de precizalla por $100 \mathrm{~s}$

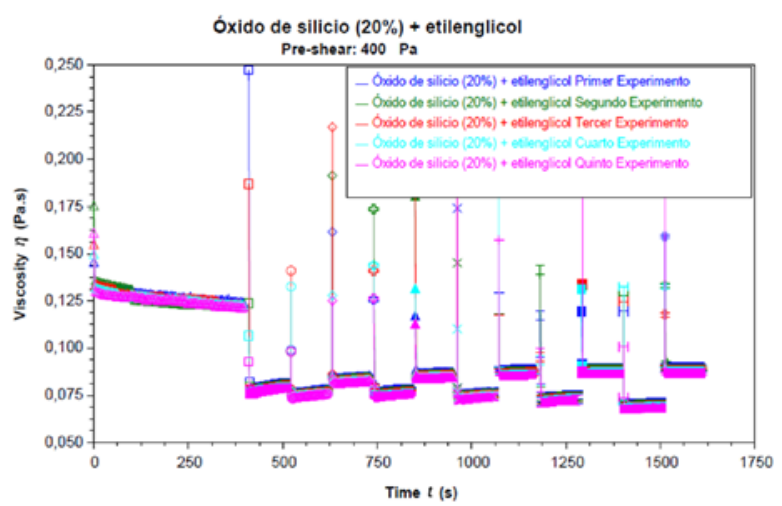

Fig. 6. Saltos de Escalón con control de esfuerzos de $\mathrm{SiO}_{2} 20 \%$ w/w con $400[\mathrm{~Pa}]$ de precizalla por 400s

Para verificar la influencia del tamaño de partículas en la suspensión, se realizaron ensayos con y sin ultrasonido y con la corrección en la pre-cizalla, Fig. 7 y Fig. 8. Las dos gráficas presentan comportamiento repetible tras cada experimento, con una dispersión de los resultados dentro de lo esperado, menor al $10 \%$. No obstante es evidente que la sedimentación se mantiene aunque su efecto no repercute en la repetitividad. Se observa que para las muestras sin ultrasonido existe mayor variación en los resultados, entre cada corrida de escalones. Por otro lado, los experimentos con ultrasonido muestran mayor repetitividad. Se podría atribuir este resultado al proceso de sedimentación de las partículas de $\mathrm{SiO}_{2}$.

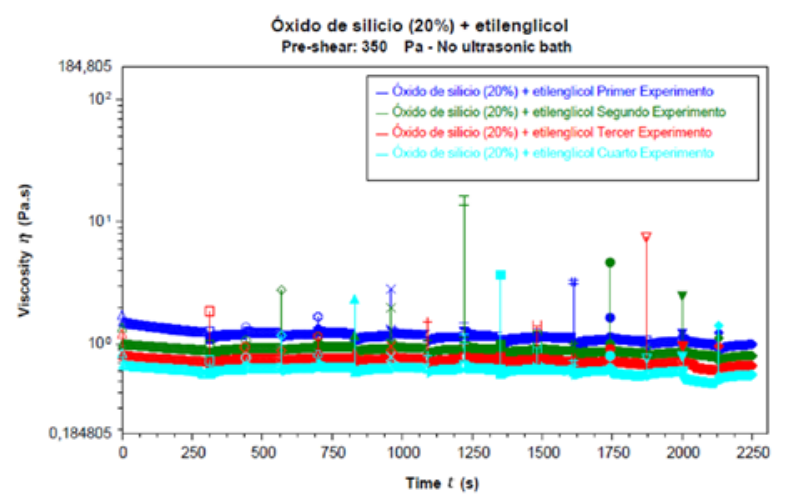

Fig. 7. Saltos de Escalón con control de esfuerzos de $\mathrm{SiO}_{2} 20 \%$ w/w con $350[\mathrm{~Pa}] \sin$ ultrasonido 


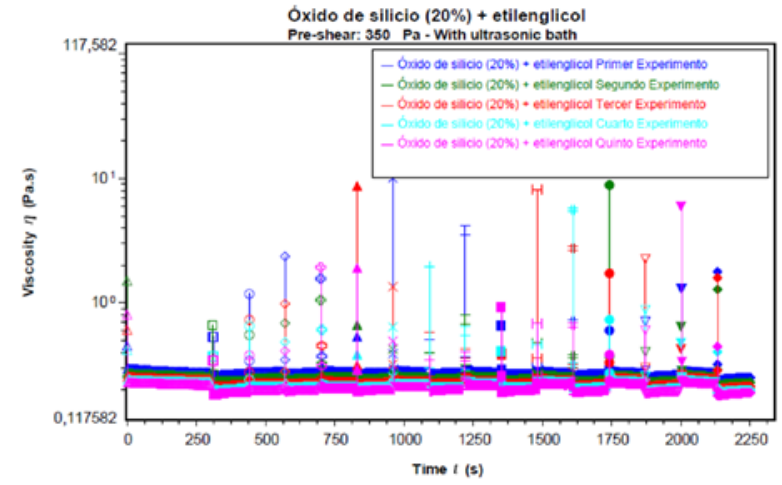

Fig. 8. Saltos de Escalón con control de esfuerzos de $\mathrm{SiO}_{2} 20 \%$ w/w con $350[\mathrm{~Pa}]$ con ultrasonido

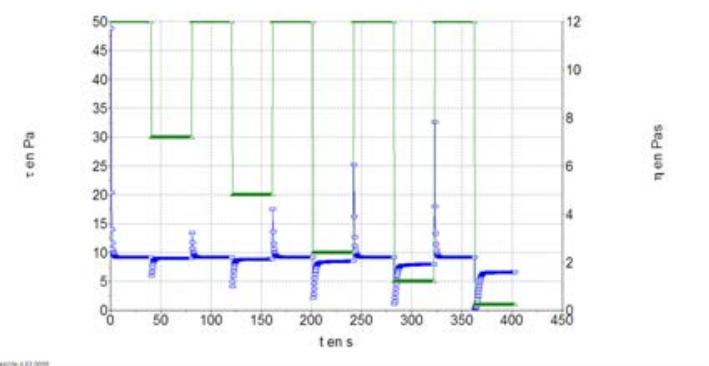

Fig. 9. Saltos de Escalón con control de esfuerzos de $\mathrm{SiO}_{2} 20 \% \mathrm{w} / \mathrm{w}$ con Reómetro Haake MarsIII

Una vez más se realizó uno de los experimentos en el laboratorio de Reología de la Universidad de Málaga para verificar su reproducibilidad. En la Fig. 9 se observa solo uno de los ensayos ya que todos se sobreponen, errores menores al 2\%. La tendencia de los resultados es la misma, por lo que hay reproducibilidad y repetitividad.

\section{CONCLUSIONES}

La respuesta reológica siempre tiene que ir acompañada de un análisis previo en el que se ponga a prueba los tres requerimientos básicos en estudios de fluidos nonewtonianos: flujo laminar, usencia de cambios físicosquímicos y repetitividad y reproducibilidad. Como se ve en este estudio la disminución de viscosidad en función de la cizalla puede ser mal-interpretada como una respuesta reológica cuando, en este caso, es causada por la sedimentación. Es importante también tener en cuenta que la estabilidad de una suspensión es, entre otros factores, dependiente de la forma y de la fracción en volumen de las partículas suspendidas. La aglomeración de partículas así como la posible absorción de fase líquida dichas partículas puede afectar notablemente la estabilidad de la suspensión. Cabe puntualizar que en este estudio se ha prescindido de componentes estabilizadores en la suspensión ya que se requería conocer el comportamiento de la suspensión en su forma más pura.

\section{AGRADECIMIENTOS}

La ejecución del presente trabajo no hubiera sido posible sin la colaboración del Laboratorio de Reología y Fluidos Complejos de la Universidad de las Fuerzas Armadas ESPE.

\section{REFERENCIAS}

[1] J. F. Morris, "A review of microstructure in concentrated suspensions and its implications for rheology and bulk flow,” Rheol. Acta, vol. 48, no. 8, pp. 909-923, Oct. 2009.

[2] W. B. Russel, "Review of the Role of Colloidal Forces in the Rheology of Suspensions,” J. Rheol. (N. Y. N. Y)., vol. 24, no. 3, pp. 287-317, Jun. 1980.

[3] W.-G. Lei and L. J. Struble, "Microstructure and Flow Behavior of Fresh Cement Paste,” J. Am. Ceram. Soc., vol. 80, no. 8, pp. 20212028, Jan. 2005.

[4] J. J. Stickel and R. L. Powell, "FLUID MECHANICS AND RHEOLOGY OF DENSE SUSPENSIONS,” Annu. Rev. Fluid Mech., vol. 37, no. 1, pp. 129-149, Jan. 2005.

[5] Y. Lee, E. Wetzel, N. W.-J. of materials science, and undefined 2003, "The ballistic impact characteristics of Kevlar ${ }^{\circledR}$ woven fabrics impregnated with a colloidal shear thickening fluid,” Springer.

[6] M. Esfahani, D. T.-J. of M. Liquids, and undefined 2017 "Experimental investigation for developing a new model for the thermal conductivity of silica/water-ethylene glycol (40\%-60\%) nanofluid at different temperatures and,” Elsevier.

[7] S. Raghavan, H. Walls, S. K.- Langmuir, and undefined 2000, "Rheology of silica dispersions in organic liquids: new evidence for solvation forces dictated by hydrogen bonding," ACS Publ.

[8] G. Żyła, J. F.-T. Acta, and undefined 2017, "Viscosity, thermal and electrical conductivity of silicon dioxide-ethylene glycol transparent nanofluids: An experimental studies,” Elsevier.

[9] L. Khiun, "Preparation and Characterization of Silicon Dioxide Nanoparticles and Thin Films," 2007.

[10] V. Gaishun, O. Tulenkova, ... I. M.-M., and undefined 2002, "Preparation and properties of colloidal nanosize silica dioxide for polishing of monocrystalline silicon wafers,” researchgate.net. 